\title{
Engajamento escolar: Efeito do suporte dos pais, professores e pares na adolescência
}

\author{
Clara Cela de Arruda Coelho'; https://orcid.org/0000-0002-8205-8542 \\ Débora Dalbosco Dell'Aglio',2; https://orcid.org/0000-0003-0149-6450
}

\begin{abstract}
Resumo
Este trabalho investigou a contribuição do suporte social da família, professores e pares para o engajamento escolar. Participaram 504 adolescentes, com idade média de 15,88 ( $\mathrm{DP}=0,88)$ matriculados no ensino médio de escolas públicas. Os instrumentos foram Questionário Sociodemográfico, Brazilian Delaware Student Engagement Scale, Social Support Appraisals e Delaware School Climate Survey. Os resultados oriundos das análises de regressão hierárquica indicaram queo suporte dos professores contribuiu de forma destacada para a explicação da variância no engajamento emocional e comportamental/cognitivo. O suporte da família se mostrou preditora penas para o engajamento comportamental/cognitivo. O suporte dos pares mostrou capacidade explicativapara ambos os tipos de engajamento, depois de considerada conjuntamente a influência do suporte da família e professores. Conclui-se que o suporte social parece influenciar de forma distinta o engajamento cognitivo/comportamental e emocional. O entendimento mais aprofundado do engajamento escolar pode favorecer a realização de intervenções melhor fundamentadas no contexto escolar.
\end{abstract}

Palavras-chave: Desempenho acadêmico; apoio social; adolescência.

\section{School Engagement: Effect of Support for Parents, Teachers, and Peers in Adolescence}

\begin{abstract}
This work investigated the contribution of the social support of the family, teachers and peers to the school engagement. Participants were 504 adolescents, with a mean age of $15.88(S D=0.88)$ enrolled in high school in public schools. The instruments were Sociodemographic Questionnaire, Brazilian Delaware Student Engagement Scale, Social Support Appraisals and Delaware School Climate Survey. Results from the hierarchical regression analysis indicated that teacher support contributed prominently to the explanation of variance in emotional and behavioral / cognitive engagement. Family support proved to be a predictor only for behavioral / cognitive engagement. Peer support showed explanatory capacity for both types of engagement, after being jointly considered the influence of support from family and teachers. We conclude that social support seems to influence cognitive / behavioral and emotional engagement differently. A more in-depth understanding of school engagement may favor the implementation of interventions that are better grounded in the school context.
\end{abstract}

Keywords: Academic Performance; social support; adolescence.

\section{Participación escolar: Efecto del soporte de los padres, profesores y pares}

\section{en la adolescencia}

\begin{abstract}
Resumen
En este estudio se investigó la contribución del soporte social de la familia, profesores y pares para la participación escolar. Participaron 504 adolescentes, con un promedio de edad de 15,88 ( $\mathrm{DP}=0,88)$ matriculados en la enseñanza secundaria de escuelas públicas. Los instrumentos fueron Cuestionario Socio-demográfico, Brazilian Delaware Student Engagement Scale, Social Support Appraisals y Delaware School Climate Survey. Los resultados derivados de los análisis de regresión jerárquica indicaron que el soporte de los profesores contribuye de forma destacada a la explicación de la variancia en el comprometimiento emocional y comportamental/cognitivo. El soporte de la familia se mostró predictor solo para el comprometimiento comportamental/cognitivo. El soporte de los pares apuntó capacidad explicativa para ambos los tipos de comprometimiento, después considerada conjuntamente la influencia del soporte de la familia y profesores. Se concluye que el soporte social parece influenciar de forma distinta el comprometimiento cognitivo/comportamental y emocional. El entendimiento más profundizado de la participación escolar puede favorecer la realización de intervenciones mejor fundamentadas en el contexto escolar.
\end{abstract}

Palabras clave: Rendimiento Académico; apoyosocial; adolescencia.

1 Universidade Federal do Rio Grande do Sul - Porto Alegre - RS - Brasil; claracela@gmail.com; dddellaglio@gmail.com

2 Universidade Lasalle - Canoas - RS - Brasil 


\section{Introdução}

O estudo do engajamento com a escola tem despertado o interesse de pesquisadores que buscam compreender formas de melhorar os resultados acadêmicos, os níveis de satisfação com a escola entre os adolescentes, assim como, diminuir as altas taxas de evasão escolar (Fredricks, Blumenfeld, \& Paris, 2004). A evasão escolar é compreendida como o estágio final do processo de desengajamento do aluno com a escola (Finn \& Zimmer, 2012). No Brasil, 45,7\% dos jovens não conseguem concluir o ensino médio até os 19 anos, dois anos depois da idade adequada,enquanto $11 \%$ dos jovens evadem da escola durante esse período (Inep, 2016). Estudantes de ensino médio que abandonam a escola tendem a enfrentar mais dificuldades na transição para a idade adulta, tais como acesso limitado a oportunidades, risco de desemprego, parentalidade precoce, dependência de terceiros e maior probabilidade de cometer crimes (Dynarski \& Gleason, 2002). Para além de consequências individuais para os próprios estudantes, altas taxas de evasão afetam a sociedade como um todo. Bons níveis educacionais garantem a criação de uma força de trabalho preparada para a concorrência com mercados mundiais, o que pode promover a economia de uma nação. Além disso, baixas taxas educacionais estão associadas ao aumento da violência e maiores custos em políticas sociais e de saúde (Kuenzer, 2010).

Além da evasão escolar, outros resultados negativos relacionados ao baixo engajamento escolar abrangem o envolvimento em delinquência, em comportamentos de risco para a saúde e em comportamentos agressivos(Carter, McGee, Taylor, \& Williams, 2007; Li \& Lerner, 2011)and school engagement, and selected health compromising and health promoting behaviours in a sample of New Zealand adolescents. Methods: A web-based survey was designed and administered to a random sample of 652 Year 11 students aged 16 years from all Dunedin (NZ. Os resultados positivos associados a melhores níveis de engajamento incluem aspectos relacionados ao desempenho acadêmico e à conclusão escolar (Jimerson, Campos, \& Jennifer, 2003) e a melhores níveis de satisfação dos estudantes com a escola, à qualidade dos relacionamentos na escola e à identificação dos alunos com a escola.

Ainda que exista unanimidade na literatura indicando que o engajamento com a escola se relaciona a uma série de resultados positivos, não há consenso sobre a definição e instrumentos de medidas do construto. De uma forma geral, o engajamento com a escola pode ser entendido como o comprometimento e envolvimento do estudante nas atividades escolares (Fredricks \& cols., 2004; Newmann, Wehlage, \& Lamborn, 1992), assim como seus sentimentos, crenças e comportamentos em relação a esse ambiente (Appleton, Christenson, Kim, \& Reschly, 2006). Em uma revisão abrangente da literatura sobre engajamento, Fredricks e cols. (2004) sugerem que há uma sobreposição considerável entre as definições de engajamento e outros construtos discutidos na literatura nas áreas de educação e psicologia, como motivação, atitude, interesse, comprometimento, apego à escola e que o engajamento pode ser melhor entendido como um meta-construto que conecta múltiplas áreas de pesquisa. Apesar de não haver uma definição única do construto, há concordância entre os autores de que o engajamento com a escola é um construto multidimensional, que compreende três domínios inter-relacionados: comportamental, emocional e cognitivo. Dessa forma, o engajamento é visto como um construto flexível e resultado de uma interação, sendo influenciado pela relação do adolescente com a escola, família e pares, indo além de uma disposição pessoal (Furlong \& cols., 2003).

O engajamento comportamental refere-se à constância do esforço, participação, assiduidade, compromisso com as tarefas escolares e outros comportamentos acadêmicos desejados (Madjar, 2017). O engajamento emocional, por sua vez, refere-se ao nível de satisfação dos estudantes com a escola, à qualidade dos relacionamentos na escola, à identificação dos alunos com a escola e ao sentimento de pertença (Sharkey, Matthew, \& Mayworm, 2014).Por fim, o engajamento cognitivo refere-se ao investimento interno por parte do próprio estudante em sua aprendizagem, à profundidade de processamento da informação e utilização de pensamento estratégico (Sharkey \& cols., 2014). Apesar de o engajamento comportamental e o engajamento cognitivo serem consideradas dimensões distintas do construto, ambos estão relacionados a melhores resultados acadêmicos, conclusão dos estudos e ajustamento emocional (Fredricks \& cols., 2004; Janosz, Archambault, Morizot, \&Pagani, 2008). Por esse motivo, neste estudo, os engajamentos comportamental e cognitivo serão avaliados conjuntamente.

Por ser um construto abrangente e multidimensional, inúmeros fatores pessoais e contextuais podem influenciar o engajamento dos alunos. Por exemplo, Sharkey, You e Schnoebelen (2008) encontraram que aspectos individuais(autoestima, valorização acadêmica dos pares, expectativas dos pais e histórico de evasão dos amigos) e aspectos relativos ao contexto escolar (segurança na escola, suporte do professor, justeza das regras) foram significativamente relacionados ao engajamento com a escola. Sharkey e cols. (2014) indicaram que ao estudar engajamento com a escola, quatro aspectos são essenciais: características individuais, relação com os pares, fatores familiares e contexto escolar no geral. Os resultados desses estudos apontam a importância de compreender como os fatores relacionados a diferentes aspectos da vida do estudante facilitam ou dificultam seu envolvimento na escola.

Embora muitos aspectos de cada contexto possam influenciar o desenvolvimento do engajamento escolar, a presença do suporte social em cada um desses contextos pode ser fundamental (Estell \& Perdue, 2013). A partir de um levantamento sobre como os estudos brasileiros têm conceituado o tema, Gonçalves, Pawlowski, Bandeira e Piccinini (2011) definem o suporte social como sendo um aspecto das relações sociais que envolve assistência material ou algum tipo de proteção emocional e que resulta em efeitos emocionais e/ou comportamentos positivos. A presença de suporte social durante a adolescência pode apresentar 
um papel destacado considerando as inúmeras mudanças físicas e psicossociais que comumente ocorrem nessa fase. Nesse período, a família, escola e amigos têm sido apontados como três principais fontes de apoio importantes para o desenvolvimento saudável (Wang \& Eccles, 2012). Estudos indicam que essas fontes de apoio têm servido como fatores protetivos, já que promovem um desenvolvimento positivo, além de reduzir aspectos negativos do desenvolvimento. No ambiente escolar, existem estudos demonstrando a importância do suporte da família e da escola (incluindo o papel dos professores) para um bom relacionamento com a escola, nos níveis acadêmicos e psicológicos, no entanto, há, comparativamente, menos informação sobre o papel do suporte dos pares para o engajamento do estudante com a escola (Estell \& Perdue, 2013; Ryan, 2010).

Neste estudo, o método para investigar a associação entre suporte social e engajamento escolar utilizado incorpora sugestões para estudos mais abrangentes ao considerar individualmentecomponentes do engajamento, a partir de duas subescalas: uma indicadora de engajamento comportamental/cognitivo e uma indicadora de engajamento afetivo(Estell \& Perdue, 2013; Fredricks \& cols., 2004). Considerar o engajamento escolar através desses indicadores pode proporcionar uma noção mais ampla do mesmo do que normalmente é fornecida por uma medida global. Partindo dessa perspectiva, o presente estudo examinou a relação preditiva entre o suporte social advindo da família, professores e pares com o engajamento comportamental/cognitivo e emocional. Mais especificamente, o suporte dos pares foi acessado após medir a influência do suporte da família e dos professoresa partir de uma regressão hierárquica. Também foi observada a relação entre os níveis de engajamento comportamental/cognitivo e afetivo e as características sociodemográficas (sexo, série, tipo de escola, repetência, configuração familiar, atividade laboral).

\section{Método}

\section{Participantes}

Participaram deste estudo 504 adolescentes, sendo $58,3 \%$ do sexo feminino, com idades entre 14 e 19 anos $(M=$ 15,88; $\mathrm{DP}=0,88)$. Os participantes estavam cursando do primeiro ao terceiro ano do ensino médio, distribuídos entre $01^{\circ}$ ano $(\mathrm{n}=242 ;$ Média de idade $=15,27 ; \mathrm{DP}=0,63), 2^{\circ}$ ano $(n=186$; Média de idade $=16,15 ; D P=0,56)$ e $3^{\circ}$ ano $(n=73$; Média de idade $=17,15$; $\mathrm{DP}=0,46$ ), em escolas da rede pública da cidade de Sobral/CE, sendo $58,5 \%$ da amostra advinda de escolas profissionais e $41,5 \%$ de escolas regulares. Dos participantes, 10,4\% trabalhavam, 8,2\% já haviam sido reprovados em alguma etapa da escolarização. Em relação à configuração familiar, 55\% residem em famílias nucleares, $25,2 \%$ em famílias monoparentais, $11,3 \%$ em famílias reconstituídas e $8,3 \%$ não residem com nenhum dos pais.

\section{Instrumentos}

Questionário Sociodemográfico: investiga as características biosociodemográficas de cada participante (sexo, série, tipo de escola, configuração familiar, situação laboral, repetência).

Brazilian Delaware Student Engagement Scale- Brazilian DSES (Holst, Weber, Bear, \& Lisboa, 2016): é composta por 11 itens respondidos por meio de uma escala do tipo Likert, variando de 1 (discordo muito) a 4 (concordo muito), distribuídos em duas subescalas que avaliam a percepção do estudante acerca de seu engajamento cognitivo/comportamental e engajamento emocional. A escala apresentou Alfa de 0,79 no estudo de adaptação para a população brasileira (Holst \& cols., 2016).

Social Support Appraisals (Vaux \& cols., 1986): escala adaptada para o Brasil por Squassoni e Matsukura (2014) que tem por objetivo verificar o suporte social percebido pelos participantes. É uma escala do tipo Likert de seis pontos que variam de "concordo totalmente" a "discordo totalmente". O estudo original apresentou boa consistência interna da escala, com coeficiente alfa de 0,74 (Squassoni \& Matsukura, 2014). Outro estudo para avaliar o suporte social em adolescentes de escolas públicas brasileiras encontrou coeficiente alfa de 0,91 (Alves \& Dell'Aglio, 2015). Para as análises do presente estudo, foram utilizadas apenas as subescalas de suporte da família e suporte dos professores.

Delaware School Climate - Versão Estudante: validado para o Brasil por Holst e cols. (2016), apresentou coeficiente alfa de 0,86. É composto por 36 itensrespondidos por meio de uma escala do tipo Likert, variando de 1 (discordo muito) a 4 (concordo muito), distribuídos em seis subescalas: relacionamento professor-estudante, relacionamento estudante-estudante, justeza das regras e clareza de expectativas, segurança na escola, bullying e engajamento estudantil. Para o presente estudo foi utilizada apenas a subescala relacionamento estudante-estudante com o objetivo de acessar a percepção do estudante do relacionamento dos pares no contexto escolar.

\section{Procedimentos}

A pesquisa foi aprovada pelo Comitê de Ética em Pesquisa do Instituto de Psicologia da UFRGS, sob o protocolo $n^{\circ}$ 1.588.302. Foram respeitados todos os aspectos éticos que garantem a integridade dos participantes, conforme previsto na Resolução 466 do Conselho Nacional de Saúde (CNS, 2012). Foi realizado o contato com a Secretaria Estadual de Educação do Ceará a fim de expor os objetivos da pesquisa esolicitar autorização para a coleta de dados nas escolas. A seguir, o projeto de pesquisa foi apresentado nas escolas participantes e foi obtida a assinatura do Termo de Concordância de cada escola. As seis escolas públicas foram selecionadas por conveniência, em diferentes regiões da cidade de Sobral. Foi solicitado o Termo se Consentimento Livre e Esclarecido (TCLE) para os pais ou cuidadores e o 
Termo de Assentimento para os adolescentes que concordaram em participar. A aplicação dos instrumentos foi realizada de forma coletiva e durou em média 30 minutos.

\section{Análise dos Dados}

Através do software SPSS (versão 21), realizaram-se testes $t$ de student e ANOVA para comparar as médias de engajamento (comportamental/cognitivo e emocional) em diferentes subgrupos da amostra (sexo, ano escolar, tipo de escola, configuração familiar, situação laboral e repetência). Em seguida, calcularam-se os tamanhos de efeito entre as médias dos grupos em que houve diferença estatisticamente significativa (Cohen, 1988). Foram também observadas as médias, desvios-padrão e correlações de Pearson para cada variável de interesse. Posteriormente, foram testados modelos de regressão hierárquica, tendo como variáveis explicativas o suporte da família, professores e relacionamento entre pares e variáveis de desfecho os engajamentos comportamental/cognitivo e emocional.

\section{Resultados}

Em relação às características sociodemográficas, não houve diferenças estatisticamente significativas entre os tipos de engajamento entre homens e mulheres, nem entre os estudantes que trabalham e não trabalham. Também não houve diferenças entre os tipos de engajamento em relação à configuração familiar. Os resultados são apresentados na Tabela 1.

Foi observada diferença estatisticamente significativa nos níveis de engajamento comportamental/cognitivo e emocional entre os alunos das escolas profissionais e regulares, com tamanhos de efeito médio e pequeno, respectivamente. Também foi observada diferença significativa no engajamento comportamental/cognitivoentre os alunos que já repetiram de ano ao menos uma vez, e os que não repetiram, com tamanho de efeito médio. Não houve diferença significativa entre os alunos que já repetiram de ano ao menos uma vez e os que não repetiram para o engajamento emocional.

Tabela 1. Diferenças de Média no engajamento comportamental/cognitivo e emocional em diferentes condições.

\begin{tabular}{|c|c|c|c|c|}
\hline \multirow[b]{2}{*}{ Condição } & \multicolumn{2}{|c|}{ Engajamento Compt/Cognitivo } & \multicolumn{2}{|c|}{ Engajamento Emocional } \\
\hline & $M(D P)$ & $t / F, p$ ed & $M(D P)$ & $t / F, p$ ed \\
\hline Homem & $15,72(2,24)$ & \multirow{2}{*}{$0,34(p=0,73)$} & $15,66(2,70)$ & \multirow{2}{*}{$1,14(p=0,25)$} \\
\hline Mulher & $15,79(2,20)$ & & $15,39(2,68)$ & \\
\hline Trabalha & $15,57(2,46)$ & \multirow{2}{*}{$0,60(p=0,54)$} & $15,42(2,68)$ & \multirow{2}{*}{$1,62(p=0,10)$} \\
\hline Não Trabalha & $15,77(2,18)$ & & $16,03(2,56)$ & \\
\hline Escola Regular & $15,16(1,99)$ & \multirow{2}{*}{$5,05^{\star *}(p<0,001 ; d=0,47)$} & $15,17(2,59)$ & \multirow{2}{*}{$2,39^{*}(p=0,02 ; d=0,21)$} \\
\hline Escola Profissional & $16,19(2,36)$ & & $15,73(2,60)$ & \\
\hline Repetiu de ano & $14,65(2,25)$ & \multirow{2}{*}{$3,33^{* *}(p=0,01 ; d=0,54)$} & $15,05(2,28)$ & \multirow{2}{*}{$1,15(p=0,25)$} \\
\hline Não Repetiu & $15,85(2,17)$ & & $15,54(2,63)$ & \\
\hline $1^{\circ}$ ano & $15,83(2,16)$ & \multirow{2}{*}{$5,48^{\star *}(p=0,004)$} & $15,49(2,46)$ & \multirow{2}{*}{$5,17^{* *}(p=0,006)$} \\
\hline $2^{\circ}$ ano & $15,99(2,23)$ & & $15,84(2,56)$ & \\
\hline $3^{\circ}$ ano & $15,00(2,23)$ & & $14,70(2,91)$ & \\
\hline Nuclear & $15,93(2,06)$ & & $15,52(2,56)$ & \\
\hline Monoparental & $15,72(2,53)$ & $2,12(p=0,09)$ & $15,55(2,72)$ & $0,16(p=0,91)$ \\
\hline Reconstituída & $15,14(2,05)$ & & $15,54(2,17)$ & \\
\hline
\end{tabular}

${ }^{*} p \leq 0,05,{ }^{* *} p \leq 0,01$ 
Tabela 2. Regressão Hierárquica - Engajamento Comportamental/Cognitivo.

\begin{tabular}{|c|c|c|c|c|c|}
\hline Engajamento Compt/Cognitivo & $\mathrm{B}$ & SE B & BPadronizado & $\mathrm{R}^{2}$ ajustado & $\Delta \mathrm{R}^{2}$ \\
\hline Etapa 1 & & & & $0,10^{* *}$ & \\
\hline Suporte Família & 0,10 & 0,01 & $0,31^{* *}$ & & \\
\hline Etapa 2 & & & & $0,22^{* *}$ & $0,12^{* *}$ \\
\hline Suporte Família & 0,04 & 0,01 & $0,14^{* *}$ & & \\
\hline Suporte Professor & 0,16 & 0,02 & $0,39^{* *}$ & & \\
\hline Etapa 3 & & & & $0,27^{* *}$ & $0,06^{* *}$ \\
\hline Suporte Família & 0,03 & 0,01 & $0,11^{*}$ & & \\
\hline Suporte Professor & 0,12 & 0,02 & $0,29^{* *}$ & & \\
\hline Rel. Pares & 0,25 & 0,04 & $0,27^{* *}$ & & \\
\hline
\end{tabular}

Tabela 3. Regressão Hierárquica - Engajamento Emocional

\begin{tabular}{|c|c|c|c|c|c|c|}
\hline Engajamento Emocional & & B & SE B & B Padronizado & $\begin{array}{l}\mathrm{R} \\
\text { ajustado }\end{array}$ & $\Delta \mathrm{R}^{2}$ \\
\hline Etapa 1 & & & & $0,07^{*}$ & & \\
\hline Suporte Família & 0,10 & 0,02 & $0,28^{*}$ & & & \\
\hline Etapa 2 & & & & $0,34^{*}$ & $0,27^{*}$ & \\
\hline Suporte Família & 0,00 & 0,01 & 0,01 & & & \\
\hline Suporte Professor & 0,28 & 0,02 & $0,58^{*}$ & & & \\
\hline Etapa 3 & & & & $0,41^{*}$ & $0,07^{*}$ & \\
\hline Suporte Família & 0,00 & 0,01 & $-0,02$ & & & \\
\hline Suporte Professor & 0,23 & 0,02 & $0,47^{*}$ & & & \\
\hline Rel. Pares & 0,32 & 0,04 & $0,29^{*}$ & & & \\
\hline
\end{tabular}

${ }^{*} p \leq 0,01$

Houve diferença significativa, indicada por um teste Bonferroni, entre as séries escolares no engajamento comportamental/cognitivo, com o primeiro ano apresentando maior média que o terceiro ano $(p=0,015 ; d=0,37)$ e o segundo ano apresentando maior média que o terceiro ano $(p=0,004$; $\mathrm{d}=0,44)$. O tamanho de efeito observado para as diferenças entre as médias foi pequeno entre o primeiro e o terceiro ano e mediano entre o segundo e o terceiro ano. Também houve diferença significativa, com tamanho de efeito mediano entre as séries no engajamento emocional, com o segundo ano apresentando maior média que o terceiro $(p=0,004 ; d=0,41)$.

A partir de correlações de Pearson, os indicadores de engajamento (comportamental/cognitivo eemocional) com a escola foram positivamente relacionadoscom as medidas de suporte social da família, professores e relacionamento com os pares (com $\mathrm{p}<0,01$ para todas as correlações). A maior 
correlação observada foi entre o engajamento emocional e o suporte dos professores $(r=0,59)$ e a menor entre o engajamento emocional e o suporte da família $(r=0,28)$. Na Tabela 2 , são descritos indicadores referente a três etapas da regressão hierárquica múltipla, tendo como variáveis de desfecho o engajamento comportamental. $\mathrm{Na}$ etapa 1 , investigou-se a influência do suporte familiar na variável de desfecho, com $F(1,496)=54,49, p<0,01$. Na etapa 2 , adicionou-se a variável suporte dos professores ao modelo, $\operatorname{com} F(2,495)=70,15$, $p<0,01$. Em seguida, na etapa 3 , incorporou-se a variável relacionamento com os pares ao modelo, com $\mathrm{F}(3,494)=63,36$, $p<0,01$. Os três modelos mostraram-se estatisticamente significativos. O terceiro modelo, descrito na etapa 3, explicou $27 \%$ do engajamento comportamental/cognitivo.

$\mathrm{Na}$ Tabela 3, são descritos indicadores referente a três etapas da regressão hierárquica múltipla, tendo como variáveis de desfecho o engajamento emocional. Na etapa 1 , investigou-se a influência do suporte familiar na variável de desfecho, com $F(1,496)=40,83 ; p<0,01$. Na etapa 2, adicionou-se a variável suporte dos professores ao modelo, com $F(2,495)=131,41 ; p<0,01$. Em seguida, na etapa 3 , incorporou-se a variável relacionamento com os pares ao modelo, com $F(3,494)=116,79 ; p<0,01$. Os três modelos mostraram-se estatisticamente significativos, sendo que o modelo três explicou $41 \%$ da variação do engajamento emocional.

\section{Discussão}

O objetivo deste estudo foi investigar a relação do engajamento com a escola (comportamental/cognitivo e emocional) com o suporte social advindo da família, professores e pares, percebido pelos estudantes. A contribuição do relacionamento com os pares foi acessada depois de verificada a influência do contexto familiar (suporte social da família) e do contexto escolar (suporte dos professores), dois contextos importantes associados ao desenvolvimento do engajamento com a escola. Dessa forma, foi possível investigar como a variável relacionamento com os pares explica parte da variância no engajamento com a escola.

Em relação às características sociodemográficas investigadas no estudo, o sexo e o trabalho não se constituíramfatoresinfluentes nos níveis de engajamento escolar. Em relação à série, os alunos mais jovens obtiveram melhores níveis de engajamento que os mais velhos. Ainda que este não seja um padrão único (Li \& Lerner, 2011), estudos anteriores indicam uma diminuição no engajamento escolar durante o curso da escolarização, especificamente a partir do ensino fundamental II, estendendo-se ao ensino médio, quando de fato se observa os maiores índices de evasão escolar (Inep, 2016). Menores níveis de engajamento escolar podem ser explicados, em parte, devido ao aumento do número de professores,à medida em que aumenta a série escolar, o que tornamais difícil construir conexões próximas (Wang \& Eccles, 2012). No entanto, são necessárias maiores investigações sobre outros fatores que podem causar esse desinteresse pela escola.
Os estudantes que indicaram ter tido uma ou mais reprovações ao longo de sua escolaridade apresentaram médias mais baixas para o engajamento comportamental/ cognitivo, porém não foi observada diferença significativa quando considerado o engajamento emocional. Uma hipótese é de que a reprovação não funcione como estratégia para melhorar os níveis de engajamento, mais especificamente o engajamento comportamental e cognitivo, que está associado a melhores resultados escolares. Com efeito, os alunos que são reprovados tendem a ter mais chance de abandonar a escola, além de a repetência, comumente, não ter impacto positivo no nível de aprendizado nos anos seguintes, podendo trazer consequências negativas para a permanência na escola (Dynarski \& Gleason, 2002; Leon \& Menezes-Filho, 2003). De fato, as emoções negativas que podem ser desencadeadas pela reprovação escolar tendem a correlacionar-se à presença de um baixo senso de autoeficácia, comprometendo o engajamento dos alunos no ambiente escolar (Luszczynska, Gutierrez-Doña, \& Schwarzer, 2005). Apesar disso, a escola continua a ter uma função de socialização importante, especialmente no período da adolescência, o que pode favorecer a permanência destes alunos na escola.Além disso, para jovens de menor renda, a escola é vista como um local mais valorizado por possibilitar acesso a recursos que não teriam em suas residências (Marques, Dell'Aglio, \& Sarriera, 2009). Ainda, houve diferença significativa entre os tipos de escola pesquisadas. As escolas profissionais obtiveram melhores médias para os engajamentos comportamental/cognitivo e emocional. Em relação ao público das escolas regulares, as escolas profissionais apresentam, no geral, menores índices de reprovação, evasão e distorção idade/série e melhores notas no ensino fundamental (Inep, 2016; Governo do Estado do Ceará, 2016). Tais fatores são indicativos de melhores níveis de engajamento (Dynarski \& Gleason, 2002; Green \& cols., 2012). No entanto, mais estudos, que incluam métodos qualitativos, podem fornecer uma avaliação mais aprofundada dos contextos escolares que ajudem a entender essas diferenças.

Em relação à investigação acerca da influência dos diferentes tipos de configurações familiares (famílias nucleares, monoparentais e reconstituídas), não houve diferenças para os níveis de engajamento com a escola. Tal resultado vai de encontro ao entendimento do fracasso escolardeterminado apenas pelotipo de família e aponta para a necessidade de uma análise funcional e multicontextual, em que fatores individuais, familiares e escolares interatuem (Pezzi, Donelli, \& Marin, 2016).Ademais, alguns pesquisadores têm indicado que o tipo de influência que a família acarreta não está, necessariamente, associada à sua configuração, mas sim às relações que se estabelecem entre os seus membros (Oliveira, Siqueira, Dell'Aglio, \& Lopes, 2008).

No geral, estudantes que perceberam mais suporte da família, dos professores e dos pares mostraram-se mais engajados com a escola. Mais especificamente, quando considerada conjuntamente a influência do suporte dos professores e pares, o suporte da família foi um preditor significativo apenas para o engajamento comportamental/ 
cognitivo. Outros estudos (Anderson, Sabatelli, \& Kosutic, 2007; Wang, Selman, Dishion, \& Stormshak, 2010) encontraram que adolescentes filhos de pais que provêm suporte são mais propensos a engajar-se em atividades pró-sociais, participar ativamente na escola e evitar problemas na escola, sendo que tais medidas relacionam-se ao engajamento comportamental. Ainda, o menor peso que o suporte da família obteve para ambos os tipos de engajamento, se comparado ao suporte dos professores e pares, seguiu um padrão habitual encontrado nos estudos quando considerado o período da adolescência em que o suporte advindo de fontes externas à família aumenta em importância (Gerard \& Booth, 2015). Desse modo, o apoio dos professores e dos amigos tende a ganhar mais relevância nesta fase (Roeser, Eccles, \& Sameroff, 2000).

Ao analisar o suporte advindo dos professores, verificou-se que essa variável apresentou a maior contribuição na explicação da variância total nos modelos em que foi inserido, para ambos os tipos de engajamento, ainda que tenha obtido um peso maior (observado pelo valor de b) quando se considerou o engajamento emocional com a escola. Esse resultado está de acordo com pesquisas que demonstraram que os alunos apresentam mais engajamento com a escola quando os professores oferecem suporte, que inclui atitudes como proximidade, aceitação, ajuda, orientação (Estell \& Perdue, 2013), suporte para a autonomia (Klem \& Connell, 2004). Tais estudos indicam que a qualidade do relacionamento com os professores age como uma "cola psicológica", que conecta os estudantes ao ambiente escolar, fornecendo uma base para o desenvolvimento do engajamento do estudante com a escola. Com efeito, este estudo sugere que o fator que parece ter mais influência no engajamento escolar é o suporte que os alunos percebem de seus professores, resultado já encontrado em outros estudos (Gruman, Harachi, Abbott, Catalano, \& Fleming, 2008; Wang \& Eccles, 2012).

O relacionamento com os pares contribuiu para a variância de ambos os tipos de engajamento após considerada a variância compartilhada com as outras variáveis (suporte da família e suporte dos professores). Outros estudos observaram essa mesma relação entre a presença de relacionamento de suporte com os pares e o engajamento comportamental e emocional (Garcia-Reid, 2007, Wentzel \& Muenks, 2016). Apesar de poucos estudos terem investigado o papel do suporte social dos pares no engajamento cognitivo, há evidências que sugerem que os adolescentes desenvolvem maior confiança e competência acadêmica quando discutem seus pontos de vista e criticam o trabalho dos colegas e quando sentem-se aceitos por seus pares (Guthrei \& Wigfield, 2000). Tal resultado está de acordo com evidências que apontam a relevância da influência dos pares durante a adolescência. Alguns estudos encontraram que o suporte dos pares estaria mais relacionado ao engajamento afetivo (Estell \& Perdue, 2013). A falta de consenso nessa questão pode ser explicada, em parte, pelas variadas formas de medir os tipos de engajamento (Fredricks \& cols., 2004).

\section{Considerações Finais}

Depois de acessar o papel do suporte da família, dos professores e dos pares para o engajamento com a escola, foi possívelidentificar que diferentes fontes de suporte social não tiveram o mesmo impacto sobre o engajamento dos adolescentes, ou seja, seu efeito diferiu entre os tipos de engajamento investigados. Os resultados do estudo sugerem que o suporte dos professores desempenhou um papel central para ambos os tipos de engajamento. No entanto, o suporte da família foi mais importante para o engajamento comportamental/cognitivo, enquanto que não acrescentou explicação para o engajamento emocional com a escola quando considerados a influência dos professores e pares. Ainda, a percepção de um relacionamento positivo entre os pares foi importante mesmo quando considerada a influência da família e dos professores para os tipos de engajamento pesquisados.

Com relação às limitações deste estudo, aponta-se que as medidas utilizadas para avaliar o engajamento escolar (comportamental/cognitivo e emocional) e o suporte social englobaram três instrumentos de autorrelato padronizados, com respostas fechadas, aplicados em um único momento no tempo. Tal metodologia pode vir a desconsiderar outras possíveis variáveis intervenientes, tendo em vista que o construto do engajamento com a escola é complexo e multideterminado. Estudos longitudinais, que possibilitem a observação das variações do engajamento ao longo da adolescência, podem trazer contribuições importantes para a compreensão desse construto. Além disso, estudos que levem em consideração fontes diversas de informação dentro do contexto escolar, como a observação de notas, taxa de frequência e percepções de outros atores do contexto escolar (professores, direção e pais), por exemplo, podem conferir maior objetividade aos dados.

\section{Referências}

Alves, C. F.; Dellaglio, D. D. (2015). Percepção de apoio social de adolescentes de escolas públicas. Revista de Psicologia da IMED, 7, 89-98.doi:10.18256/2175-5027/psico-imed.v7n2p89-98

Anderson, S. A.; Sabatelli, R. M.; Kosutic, I. (2007). Families, urban neighborhood youth centers, and peers as contexts for development. Family Relations, 56, 346-357. doi:10.1111/j.17413729.2007.00464.x

Appleton, J. J.; Christenson, S. L.; Kim, D.; Reschly, A. L. (2006). Measuring cognitive and psychological engagement: Validation of the Student Engagement Instrument. Journal of School Psychology, 44(5), 427-445. doi:10.1016/j.jsp.2006.04.002

Carter, M.; McGee, R.; Taylor, B.; Williams, S. (2007). Health outcomes in adolescence: Associations with family, friends and school engagement. Journal of Adolescence, 30(1), 51-62. doi:10.1016/j. adolescence.2005.04.002 
Conselho Nacional de Saúde[CNS] (2012). Resoluçãon n 466, de 12 de dezembro de 2012.Recuperado: 10 mai. 2016. Disponível: http:// www.sap.sp.gov.br/download_files/pdf_files/comite_de_etica_ em_pesquisa_SAP/resolucao-466_12-12.pdf.

Cohen, J. (1988). Statistical power analysis for the behavioral sciences (2nd ed.). Hillsdale, NJ: Lawrence Earlbaum Associates.

Dynarski, M.; Gleason, P. (2002). How we can help? What we have learned from recent federal dropout prevention evaluations. Journal of Education for Student Placed At Risk, 7(1), 43-69.doi:1 $0.1080 / 10824669.2011 .545977$

Governo do Estado do Ceará (2016). Educaçãoprofissional. SEDUC Secretaria da Educação, Governo do Estado do Ceará.Disponível: http://educacaoprofissional.seduc.ce.gov.br

Estell, D. B.; Perdue, N. H. (2013). Social support and behavioral and affective school engagement: The effects of peers, parents, and teachers. Psychology in the Schools, 50(4), 325-339. doi:10.1002/ pits. 22053

Finn, J. D.; Zimmer, K. S. (2012). Student engagement: What is it? Why does it matter? In: Christenson, S. L.;Reschly, A. L.; Wylie,C. (Orgs.), Handboof of Research on School Engagement (1st ed., pp. 97-131). New York: Springer.

Fredricks, J. A.; Blumenfeld, P. C.; Paris, A. H. (2004). School Engagement: Potential of the Concept, State of the Evidence. Review of Educational Research, 74(1), 59-109. doi:10.3102/00346543074001059

Furlong, M. J.; Whipple, A. D.; Jean, G.; Simental, J.; Soliz, A.; Punthuna, S. (2003). Multiple contexts of school engagement: Moving toward a unifying framework for educational research and practice. California School Psychologist, 8, 99-113. doi:10.1007/ BF03340899

Garcia-Reid, P. (2007). Examining social capital as a mechanism for improving school engagement among low income Hispanic girls. Youth \& Society, 39, 164-181. doi:0.1002/9781119085621. wbefs 430

Gerard, J. M.; Booth, M. Z. (2015). Family and school influences on adolescents' adjustment: The moderating role of youth hopefulness and aspirations for the future. Journal of Adolescence, 44, 1-16. doi:10.1016/j.adolescence.2015.06.003

Gonçalves, T. R.; Pawlowski, J.; Bandeira, D. R.; Piccinini, C. A. (2011). Avaliação de apoio social em estudos brasileiros: aspectos conceituais e instrumentos. Ciência \& Saúde Coletiva, 16(3), 155169.doi:10.1590/S1413-81232011000300012

Green, J.; Liem, G. A. D.; Martin, A. J.; Colmar, S.; Marsh, H. W.; Mclnerney, D. (2012). Academic motivation, self-concept, engagement, and performance in high school: Key processes from a longitudinal perspective. Journal of Adolescence, 35(5),
111-1122. doi:10.1016/j.adolescence.2012.02.016

Gruman, D.; Harachi, T. W.; Abbott, R. D.; Catalano, R. F.; Fleming, C. B. (2008). Longitudinal effects of student mobility on three dimensions of elementary school engagement. Child Development, 79(6), 1833-1852. doi:10.1111/j.1467-8624.2008.01229.x

Guthrei, J. T.; Wigfield, A. (2000). Engagement and motivation in reading. In: Kamil, M. L.; Mosentahl, P. B.;Pearson, P. D.; Barr,D. (Orgs.), Handobook of reading research (3ed ed., pp. 403-422). New York: Longman.

Holst, B.; Weber, J. L.; Bear, G. G.; Lisboa, C. S. M. (2016). Brazilian cross-cultural adaptation and content validity of the Delaware School Climate Survey-Student (DSCS-). RELIEVE: Revista Electronica de Investigacion Y Evaluacion Educativa, 22, 1-12. doi:10.7203/relieve.22.2.6459

Institudo Nacional de Estudos e Pesquisas Educacionais Anísio Teixeira [INEP] (2016). Censo escolar da educação básica 2016. Brasil: MEC. Disponível: http://download.inep.gov.br/educacao_ basica/censo_escolar/notas_estatisticas/2017/notas_estatisticas_ censo_escolar_da_educacao_basica_2016.pdf

Janosz, M.; Archambault, I.; Morizot, J.; Pagani, L. S. (2008). School engagement trajectories and their differential predictive relations to dropout. Journalof Social Issues, 64(1), 21-40. doi: 10.1111/j.15404560.2008.00546.x\}

Jimerson, S. R.; Campos, E.; Jennifer, L. G. (2003). Toward an understanding of definitions and measures of school engagement and related terms. The California School Psychologist, 8(1), 7-27. doi:10.1007/BF03340893

Klem, A. M.; Connell, J. P. (2004). Relationships matter: Linking teacher support to student engagement and achievement. The Journal of School Health, 74, 262-273.

Kuenzer, A. Z. (2010). O ensino médio no Plano Nacional de Educação 2011-2020: Superando a década perdida? Educação \& Sociedade, 31(112), 851-873. doi:10.1590/S0101-73302010000300011

Leon, F. L. L.; Menezes-Filho, N. A. (2003). Reprovação, avanço e evasão escolar no Brasil. Pesquisa e Planejamento Econômico, PPE, 32, 417-451.

Li, Y.; Lerner, R. M. (2011). Trajectories of school engagement during adolescence: Implications for grades, depression, delinquency, and substance use. Developmental Psychology, 47, 233-247.

Luszczynska, A.; Gutierrez-Doña, B.; Schwarzer, R. (2005). General self-efficacy in various domains of human functioning: Evidence from five countries. Psychology Press, 40(2), 80-89.

Madjar, N. (2017). Stability and change in social goals as related to goal structure and engagement in school. Journal of Experimental Education, 85(2), 259-277. 
Marques, L. F.; Dell'Aglio, D. D.; Sarriera, J. S. (2009). O tempo livre na juventude brasileira. In: Libório, R. M. C.; Koller, S. H. (Orgs.), Adolescência e juventude: Risco e proteção na realidade brasileira (pp. 79-106). São Paulo: Casa do Psicólogo.

Newmann, F.; Wehlage, G. G.; Lamborn, S. D. (1992). The significance and sources of student engagement. In: Newmann,F. (Org.), Student engagement and achievement in American secondary schools (pp. 11-39). New York: Teachers College Press.

Oliveira, D.; Siqueira, C. S.; Dell'Aglio, D. D.; Lopes, R. C. S. (2008). Impacto das configurações familiares no desenvolvimento de crianças e adolescentes: Uma revisão da produção científica. Interação Em Psicologia, 12(1), 87-98.

Pezzi, F. A. S.; Donelli, T. M. S.; Marin, A. H. (2016). School failure in the perception of adolescents, parents and teachers. Psico-USF, 21, (2), 319-330. doi:10.1590/1413-82712016210209

Roeser, R. W.;Eccles, J. S.; Sameroff, A. J. (2000). School as a context of early adolescents academic and social-emotional development: A summary of research findings. The Elementary School Journal, 100(3), 443-471.

Ryan, A. M. (2010). Peer groups as a context for the socialization of adolescents' motivation, engagement, and achievement in school. Educational Psychologist, 35(2), 125-141. doi:10.1207/ S15326985EP3502

Sharkey, J. D.; Matthew, Q.; Mayworm, A. M. (2014). Student engagement. In: Furlong, M. J.; Gilman, R.; Huebner,E. S. (Orgs.), Handbook of Positive Pshychology in Schools (2nd ed., pp. 176192). New York.
Sharkey, J. D.; You, S.; Schnoebelen, K. (2008). Relations among school assets, individual resilience, and student engagement for youth grouped by level of family functioning. Psychology in the Schools, 45(5), 402-418.doi:10.1002/pits.20305

Squassoni, C. E.; Matsukura, T. S. (2014). Adaptação transcultural da versão portuguesa Social Support Appraisals para o Brasil. Psicologia: Reflexão E Crítica, 27(1), 1-10.doi:10.1590/S010279722014000100009

Vaux, A.; Philips, J.; Holly, L.; Thompson, B.; Williams, D.; Stewart, D. (1986). The Social Support Appraisals (SSA) Scale: Studies of reliability and validity. American Journal of Community Psychology, 14, 195-220.

Wang, M.; Eccles, J. (2012). Social support matters: Longitudinal effects of social support of three dimensions of school engagement from middle to high school. Child Development, 83(3), 877-895. doi:10.1111/j.1467-8624.2012.01745.x.

Wang, M. T.;Selman, R. L.; Dishion, T. J.; Stormshak, E. A. (2010). A tobit regression analysis of the covariation between middle school students' perceived school climate and problem behavior. Journal of Research on Adolescence, 20, 274-286.

Wentzel, K. R.; Muenks, K. (2016). Peer influence on students'motivation, academic achievement, and social behavior. In: Wentzel,K. R.; Ramani,G. B. (Orgs.), Handbook of social influences in school contexts (pp. 13-30). New York: Routledge.

Recebido: 12 de janeiro de 2017 Aceito: 24 de setembro de 2018

As autoras agradecem à Coordenação de Aperfeiçoamento de Pessoal de Nível Superior (CAPES) pela bolsa de Mestrado concedida à primeira autora. unrestricted use, distribution and reproduction in any medium, provided the original article is properly cited. 